\title{
Joutes de freestyle - faut-il rimer ou bien se moquer?
}

\author{
Ricardo Indig Teperman \\ Universidade de São Paulo
}

Le freestyle est une variante du rap, marquée par le fait qu'elle est (ou au moins semble) improvisée. Ces improvisations se présentent souvent sous forme de 'joute' ou de 'bataille', comme celles qui ont lieu depuis cinq ans, tous les samedis soirs, à la sortie du métro Santa Cruz, à São Paulo . Les jeunes qui ont créé cette pratique et qui s'occupent encore aujourd'hui de sa continuité se sont organisés en un collectif appelé : Afrika Kidz Crew².

L'événement attire, en moyenne, cinquante personnes chaque semaine: quelques femmes mais surtout des jeunes gens, qui ont entre quinze et vingt-cinq ans, la plupart venant de familles défavorisées ${ }^{3}$. La majorité des habitués de Santa Cruz se lance dans des improvisations de rimes et, dans ce but, les spectateurs et les protagonistes de la joute ne forment plus qu'un seul groupe. De plus, leur distribution dans l'espace n'établit pas non plus de démarcation très claire entre la scène et le public. Ils ne se rangent pas vraiment en cercle, mais plutôt comme s'ils se tassaient dans un coin - ici, devant le mur d'un célèbre lycée catholique. Il n'y a ni système de sonorisation, ni

1 J'accompagne les joutes du métro Santa Cruz depuis juin 2008. J'ai été présent à une trentaine de rencontres, la moitié a été enregistrée en format audio et ensuite retranscrite. Ce matériel a été utilisé dans ma thèse de master, actuellement en phase de conclusion sous la direction de la Professeure Dre Lilia Schwarcz.

2 Le terme crew est utilisé pour désigner un groupe de personnes qui travaillent sur des projets communs, autour du rap. Il peut rassembler entre autres des MCs, DJs, et des producteurs. Après plusieurs remaniements au cours de ces dernières années, les MCs Julio DFlow, Lucas LTA, Marcello Gugu et Marcos Vinicius Bitrinho font partie aujourd'hui de l'Afrika Kidz Crew. Il est très courant que les MCs adoptent des surnoms plutôt que d'utiliser leur prénom civil.

3 L'affirmation se base sur des informations collectées sur le terrain comme, par exemple, le fait que la plupart des participants étudie ou bien a étudié dans des écoles publiques, dispose de très peu d'argent pour des activités de loisir, et vit dans des quartiers pauvres (comme le M’Boi Mirim) ou dans des banlieues ouvrières (comme Diadema et Santo André). 
d'estrade et ni de scène. Il arrive que, de temps en temps, les organisateurs apportent un petit amplificateur, avec lequel ils lancent des « beats " pour que les MCs rappent. Mais, la plupart du temps, les improvisations sont faites a cappella. Sur une surface qui ne fait qu'une trentaine de mètres carrés, devant la sortie des escaliers du métro, sans aucune indication du fait que depuis cinq ans, tous les samedis soirs, cet endroit devient un lieu de rencontre de jeunes venus des quatre coins de la ville. Vers huit heures du soir, de petites bandes commencent à se rassembler, pour discuter (souvent de rap), se lancer des défis rimés ou juste pour des séances de freestyle. La joute commence un peu avant vingt-deux heures et, vers minuit, presque tout le monde est déjà parti. En général, les plus jeunes rentrent chez eux, tandis que les plus âgés vont faire la fête, souvent dans un club où il y a un DJ ou un concert de rap.

Quand un des membres de l'Afrika Kidz Crew arrive, tout en prenant le temps de discuter avec ses amis, il commence à recueillir les inscriptions des MCs qui veulent participer à la bataille (en général de huit à seize personnes). Généralement, le format est le suivant: les MCs s'affrontent au cours d'étapes éliminatoires, définies par un tirage au sort réalisé en public. Chaque joute peut durer jusqu'à trois tours de trente secondes chacun, appelés : " rounds ". Avant le début de chaque duel, les défiants jouent à pair ou impair ${ }^{4}$ pour décider qui commencera. Celui qui gagne le round, demandera à l'autre de commencer le suivant, car d'après eux, il est préférable de " répliquer » que « d'attaquer $»^{5}$.

A Santa Cruz, le public a le pouvoir de juger les improvisations. A la fin de chaque round, un membre de l'Afrika Kidz, chargé de présenter la bataille ${ }^{6}$, demande aux spectateurs de faire " du bruit » pour son MC préféré. Ceux qui gagnent les étapes éliminatoires devront s'affronter lors de demies finales, d'où sortiront les deux finalistes. A l'origine, en 2006, chaque personne devait donner une participation d'un réal brésilien - la somme totale

4 Equivalent $\mathrm{du}$ " pile ou face " brésilien.

5 Celui qui termine le premier round sera obligatoirement le premier à rimer lors de la prochaine étape. Selon leur propre discours, " celui qui a terminé, commence ". S'il y a un troisième round, alors on refait un tirage à " pair ou impair ".

6 Le rôle du présentateur est d'appeler les MCs et de dire qui va rencontrer qui. A l'aide d'un assistant qui surveille le temps, il doit aussi indiquer le début et la fin de chaque improvisation. Il est, en quelque sorte, un maître de cérémonie, qui doit animer la soirée. S’il faut présenter les règles de la dispute, ce sera à lui de le faire. Et si quelqu'un enfreint une règle, c'est aussi à lui de faire la remarque. 
étant alors offerte au vainqueur. Mais, comme il arrive parfois que les meilleurs MCs ne pouvaient participer, car ils n'avaient pas d'argent, l'organisation a décidé de rendre l'inscription gratuite et d'offrir comme prix un objet choisi chaque semaine par un des membres ${ }^{7}$

L'enjeu, dans une joute, d'après les participants, c'est de « se moquer de l'autre ". Lors d'un entretien publié dans le magazine Revista Língua Portuguesa, le DJ Babão, l'un des organisateurs de la Batalha do Real, à Rio de Janeiro, explique, avec une expression typiquement carioca, quel est le but de la dispute: "déconner avec l'adversaire " ${ }^{8}$.

La stratégie rhétorique la plus efficace d'un improvisateur pour gagner une joute est l'humour. Ceux qui arrivent à faire rire le public ont de bonnes chances de gagner. On remarque que les duels le plus souvent dits " mémorables ", ainsi que les vers les plus reconnus, sont ceux qui ont le plus amusé le public. Pour illustrer l'importance de l'humour dans les duels et surtout ceux de Santa Cruz, je reproduis une interprétation indigène de la pratique, que j'ai pu enregistrer le 5 juin 2010. À la fin d'une des premières disputes de la nuit, un jeune homme, apparemment surpris par le ton moqueur des improvisateurs, a posé la question suivante: " mais faut-il rimer ou bien se moquer? " A ce moment, Julio DFlow, présentateur de la bataille de ce soir-là a répondu sans hésiter: «les deux ».

Ce constat nous autorise à penser les joutes de freestyle comme une variation contemporaine et urbaine d'un genre de pratique très courant dans certaines sociétés au cours des siècles. Johan Huizinga mentionne des jeux d'improvisation verbale des plus variés dans des traditions du monde entier. Ce sont des tournois de jurons, des concours de vantardise, des défis de rimes. Il reprend un exemple classique, tiré de Malinowski: « chez les indigènes Trobriand, on trouve des formes intermédiaires entre les tournois de vantardise et les compétitions de richesse " (Huizinga 2008: 75). Un autre auteur mentionne la globalisation du phénomène. Il s'agit de Peter Burke, qui dit que « en Sicile, au XVII ${ }^{\text {ème }}$ siècle, il y avait des défis d'improvisation qui faisait penser aux stevleik de Norvège ou bien aux disputes de haiku au Japon » (Burke 1989: 167). Dans l'étude de Roger Abrahams, publiée au début

7 Exemples de prix : CDs ou DVDs de groupes de rap, en général des copies pirates.

8 L'expression en brésilien est " tirar onda com o rival ». Le texte est disponible sur internet, à l'adresse suivante : http://revistalingua.uol.com.br/textos.asp?codigo=11478 
des années soixante sous le nom de Deep Down in the Jungle- Negro narrative folklore from the streets of Philadelphia, on trouve toute une série de documents rassemblés par l'auteur, qui suggèrent l'importance de ce type de pratique chez les afro-américains de la région.

Verbal contest accounts for a large portion of the talk between members of this group (black men in Camigerly). Proverbs, turns of phrase, jokes, almost any manner of discourse is used, not for purposes of discursive communication but as weapons in verbal battle. Any gathering of the men customarily turns into "sounding», a teasing or boasting session". (Abrahams 1970: 45) ${ }^{9}$

Encore aujourd'hui au Brésil, plusieurs modalités de défis chantés sont pratiquées, comme le cururu, la embolada, le partido-alto et le repente. Dans la rubrique desafio (défi), du Dicionário Musical Brasileiro, de Mario de Andrade, on trouve que le style où "l'un parle, l'autre répond ", est plus ou moins universel. Ce qui caractérise la version brésilienne c'est « le sport de s’insulter, souvent thème unique entre les défiants Nordestins " (Andrade 1989: 186). Dans son étude sur le cururu à Piracicaba, Allan Paula de Oliveira cite un vers du cantador Zico Moreira: " pour que le cururu soit beau, il faut qu'il frappe ". Puis, l'auteur dit:

Ces vers sont une synthèse du " canto de pau trocado ", modalité selon laquelle on provoque un autre cantador, avec des bouffonneries et des plaisanteries. "Il faut frapper ", disent les cantadores, et le prestige d'un cantador est plus grand dans la mesure où il ne se prive ni de heurter, ni de se laisser heurter (Oliveira 2007: 328) ${ }^{10}$.

L'usage du verbe "frapper " est aussi courant chez les MCs. Souvent, avant le début d'un round, un des spectateurs ou bien le présentateur provoque les improvisateurs avec des phrases comme: "vas-y, frappe fort! "; " mets-lui un couteau sur la gorge! "; " arrache lui la tête! »; " du sang! »; entre autres. Pendant le duel, les images violentes employées par les improvisateurs sont à l'origine de réactions du même type que les plaisanteries et les provocations. Le public rit, fait du bruit, se bouscule. On pourrait dire

9 L'auteur parle aussi des jeux des dozens et du signifyin'. La documentation sur les pratiques de moquerie des jeux d'improvisation rimés est très volumineuse et plusieurs auteurs suggèrent que ces jeux sont à l'origine de ce qui s'est établi comme le chant-parlé caractéristique du rap. Voir Toop 2000 et Rose 1994, entre autres.

10 Il ne s'agit pas de discuter les éventuelles origines brésiliennes des joutes de freestyle. Les exemples cités servent simplement à renforcer la comparaison générale. L’idée étant que toutes les pratiques citées peuvent être considérées comme différentes modalités de tournois d'insultes. 
que les menaces d'agression physique fonctionnent exactement comme des canulars, ce qui nous permet de reprendre la réflexion sur les relations entre l'humour et la violence. Peter Gay affirme que « le fait que la parole commune établisse des liens entre l'humour et des actes belligérants, comme mordre, percer, couper, est très révélateur. En utilisant des matériaux de sa culture, l'humour offre des opportunités splendides pour l'exercice - et le contrôle - de l'agression " (Gay 1995: 371).

Les chercheurs sur l'humour suggèrent l'existence d'un paradigme qui orienterait une bonne partie de la réflexion occidentale sur le sujet, sur l'existence de deux types de rire, le bon et le mauvais. De manière très abrégée, Georges Minois suggère que ce paradigme a été établi par des penseurs du Moyen-âge, qui disaient que le bon rire « exprime la joie du chrétien et doit être modéré, presque silencieux, presque un sourire ». Le mauvais rire serait celui de la dérision, de la plaisanterie et en même temps, un rire physique, bruyant, peu gracieux, qui secouerait le corps (Minois 2003: 232). Il montre l'intromission du problème dans les débats théologiques, paraphrasant Saint Thomas d'Aquin, qui approuvait les jeux et les divertissements, à partir du moment où ils étaient modérés. D’après Minois, la moquerie serait pour Saint Thomas « un pêché encore plus grave que l'audace, puisqu'elle humilie, cherche à faire rougir. Se moquer du mal n'est pas un bien, parce que le mal doit être pris au sérieux " (Minois 2003: 235). Il est évident que chercher une solution stable ne nous intéresse pas, une solution qui fournisse des critères de classement pour distinguer le bon et le mauvais rire. C'est le fait de classer le rire comme étant acceptable ou inacceptable qui est, pour nous, anthropologiquement intéressant.

Dans les batailles de freestyle semble prévaloir l'habitude de ne prendre en considération que le " mauvais rire " - le moteur même du jeu étant de se moquer de l'autre. Mais, il existe aussi, dans ce contexte, une limite qui doit être respectée- le " mauvais rire " doit être socialement approuvé (et, dans ce sens là, il est « bon ». Pour aller un peu plus loin, on peut dire qu'encore plus que le chercheur, les improvisateurs et les organisateurs eux-mêmes se préoccupent de contrôler les limites subtiles de la frontière entre la plaisanterie et l'offense. Il faut considérer que si ces limites sont dépassées, il est possible que le duel de rimes devienne un échange de coups de poings ou, voire, devienne ennuyeux.

$\mathrm{Au} \mathrm{XVII}{ }^{\text {ème }}$ siècle déjà, le thème de la " régulation de l'humour " était 
une importante source de préoccupation pour les français, chez qui les mots d'esprit avaient une grande valeur. Georges Minois cite plusieurs manuels de " civilité » ou de " bonne conduite », qui ont été publiés à l'époque. Dans ses Réflexions sur le ridicule et sur les moyens de l'éviter, Morvan de Bellegarde nous enseigne qu'il faut être « très malin pour se moquer des autres d'après leurs défauts ou leur conduite; cela veut dire leur donner une espèce de droit de se moquer de nous à leur tour " (Bellegarde apud Minois 2003: 446). Il est intéressant de considérer que le conseil, écrit il y a plus de trois cents ans, aurait parfaitement pu faire parti d'un manuel de freestylers. D'ailleurs, le seul document de ce genre auquel j'ai eu accès est une publication indépendante et très mal produite qui s'appelle How to freestyle, write battle raps and write rap songs. Dans la rubrique « Playground Rules », l'auteur Joseph Edward Brown, explique:

There has been a lot of drama over the years during rap battles resulting in beefs, and there will be a lot more. Let's face it: some rappers can't take losing in front of a crowd, so they retaliate during or after the battle with violence. There is nothing wrong with asking your opponent if their ego can handle losing, or if you don't think they can handle a loss in front of others, withdraw and walk away. You can't come up if you get put six feet under. Usually, rap is not a personal attack on one's family of loved ones. It will basically contain material that will degrade you as a person and a rapper. But if family does come into play, remember, it's just words. And, if you feel the need to get physical after a battle, you should consider doing something else. (BROWN 2006:9)"1

Les conseils cités ci-dessus ont, pour ainsi dire, un caractère psychologique; ils sont assez génériques et presque banals. Pour résumer, ils expliquent que le moqueur ne doit pas être surpris par le fait que l'on se moque de lui. Dans le cas des batailles de Santa Cruz, on pourrait se concentrer sur un aspect qui paraît être plus vivant pour aborder le même problème. Plus que des conseils écrits, les organisateurs des joutes proposent des règles, expliquées et interprétées in loco.

Lors de mes tous premiers entretiens avec les improvisateurs, j'ai appris que depuis les années quatre-vingt-dix, quelques MCs de la région de Diadema

11 Il faut souligner le fait qu'aux États-Unis le rap occupe une place hégémonique dans l'industrie de la variété. De plus, là-bas, le rap y a été à l'origine d'affaires très violentes, comme l'assassinat des icônes Tupac Shakur et Notorius Big. En comparaison, le rap brésilien est assez amateur (aussi bien du point de vu du produit commercial que de sa potentialité belligérante). 
se battaient en duel dans des fêtes de rap. Parmi ces " pionniers ", l'un des noms les plus cités est celui de JL, qui est allé à Santa Cruz le 14 novembre 2009. Il a été reçu par les louanges des organisateurs et des autres participants. Plusieurs fois au cours de la soirée, il a été complimenté et remercié pour sa présence.

Comme c'était la première fois que « la légende JL » venait à Santa Cruz, le présentateur Marcello Gugu a voulu lui rappeler les règles du combat: « JL, je suis sûr que t'es au courant. Mon pote... freestyle comme avant : personne ne baise personne, pas de pédérastie. T’as tout compris? Personne ne nique la mère de personne; et si c'est le cas, pas besoin de le dire ici ". Ayant perdu le pair ou impair, JL a démarré le premier round, contre MC Nino ${ }^{12}$ :

JL

Alors, c'est comme ça, je dis et je vais le signer

On m'a dit de venir ici et de t'exploser

Il est où Nino, il est à la télé

Peut-être qu'il a été exterminé?

Old school, quatre-vingt-quinze, je te dis

$\mathrm{Tu}$ es grave mon ami, mon ennemi

Il y a longtemps avant tu rimais

Mais, JL est arrivé et tout de suite t'a déchiré

Fais attention, ne te la joue pas

Nino, s'il te plaît, ne flippe pas et regarde-moi ${ }^{13}$

12 La transcription des improvisations pose plusieurs questions méthodologiques. Comment établir un critère pour séparer les vers? Comment enregistrer les gestes, les regards, les petits mots dits entre les dents des spectateurs? Ce sont des problèmes qu'on ne pourra pas discuter ici, mais qui ne peuvent pas être ignorés. Étant donné que la réaction du public joue un rôle central dans le déroulement de la bataille, j'ai décidé d'inclure, toujours entre parenthèses, un mot qui indique les réactions les plus notoires. Plutôt que de créer toute une typologie pour ces commentaires, j'ai préféré tout simplement marquer « bruits " ou bien " rires ». Les enregistrements audio et/ou vidéo des joutes commentées dans cet article, ainsi que des dizaines d'autres, sont disponibles sur Youtube. Pour la bataille entre JL et Nino, allez sur: http://www.youtube.com/watch?v=4fTtM1OTAx4

Pour celles de Pri contre Basthian, allez sur: http://www.youtube.com/watch?v=fcdztLCvuHQ et http:// www.youtube.com/watch?v=WcV3tB8n4tw

13 Étant donné que la rime est l'un des aspects les plus importants pour les improvisateurs, je ne pouvais pas tout simplement ignorer ce fait lors de la traduction. J'ai donc décidé de chercher des solutions en français de façon à préserver les rimes. Évidemment, j’ai fait très attention à ne jamais changer le sens principal des vers. 
Tout de suite on remarque l'importance des rimes. La totalité des vers d'une improvisation n'est pas forcément rimée, mais la rime est toujours recherchée. Ce n'est pas pour rien que dans les duels de freestyle et dans le rap en général, le verbe " rimer " est synonyme de " chanter ». Peut-être serait-il alors plus juste de dire que " rimer » est le verbe le plus adapté pour définir l'action des MCs : ils ne chantent pas, ils ne parlent pas, ils riment.

A la différence des techniques d'improvisations traditionnelles du repente, qui imposent des formes très strictes sur lesquelles les improvisateurs doivent rimer, dans le rap, la seule détermination structurelle est qu'on rime sur le beat, sur le bum-clap. De manière abrégée, l'idée est que la scansion des vers doit être basée sur les rythmes de la batterie typique du rap, construite avec la grosse caisse ("bum ") sur les premiers et troisièmes temps, et la caisse claire (" clap ") sur les deuxièmes et quatrièmes temps de la mesure quatre-quatre ${ }^{14}$. Les rimes tombent sur le quatrième temps de chaque paire de mesure, ce qui justifie le fait qu'on écrive des vers organisés en paires. Adam Bradley formule une idée proche quand il dit que le plus courant dans le rap, ce sont des «end rhymes, those rhymes that fall on the last beat of the musical measure, signaling the end of the poetic line. Two lines in succession with end rhymes comprise in a couplet, the most common rhyme scheme in old-school rap" (Bradley 2009: 50). Les expressions old-school et new-school se rapportent aux deux grandes phases du rap: les années quatre-vingts et le début des années quatre-vingt-dix représenteraient la old-school, tandis que la fin des années quatre-vingt-dix et les années deux mille seraient la new-school. Le sujet est polémique parmi les rappeurs, ce qui nous intéresse est de voir comment JL revendique sa participation à la old-school brésilienne.

La performance de JL n'a pas bouleversé les spectateurs, et MC Nino, choisi au hasard pour disputer le duel avec lui, a commencé son improvisation par des coups durs:

14 Cette base musicale (beat) peut être réalisée par un DJ qui mixe en direct, par la simple reproduction d'une base préenregistrée, ou bien par un beat-box (un musicien qui reproduit vocalement les sons de la basse et de la batterie). A Santa Cruz, le plus souvent, les improvisations sont a cappella, mais même dans ce cas, c'est l'idée musicale du bum-clap qui dirige les improvisations. Les MCs emploient des motifs caractéristiques du style, comme si le beat était sous-entendu. Il faut prendre en considération le fait que quand les improvisations sont a cappella, il est tout à fait acceptable et même courant que les vers durent plus de quatre temps et qu'en conséquence, les couplets soient musicalement imparfaits ou irréguliers. 
NINO

Miniature, moche comme Zacarias

Tiens p'tit joufflu, tu aimes la saucisse?

Je suis le roi de l'improvisation et tu le sais

Tâche de prier et demander s'il te plaît

Comprend bien que c'est Nino qui contrôle

Joufflu t'es venu avec ton bonnet, tu vas pêcher des soles?

Tu es pêcheur mon pote? Bang jump

Tu racontes des mensonges à la Forrest Gump

Nino arrive avec des rimes, il est dangereux

Tu sais bien qu'ici je suis contagieux

Je rime avec flow et j'envoie les rafales, c'est dingue

J'arrache ta tête avec une carabine plutôt qu'avec un flingue

Dès le premier vers Nino a réussi à y concentrer un grand nombre d'offenses. Petite taille, laideur et, évocation de Zacarias, l'inoubliable personnage de la série télévisée " Trapalhões ", il a assuré d'un seul coup plusieurs points: clown, grandes dents, chauve, vieux, homosexuel (et en plus, petit et rond).

Nino a gagné le premier round avec une grande marge de points. Il faut souligner le fait que pendant les improvisations on voit souvent apparaître des vers un peu tortueux et éventuellement dépourvus de sens. Par exemple, l'expression « bang-jump » est utilisée sans que l'on puisse la comprendre. Mais, outre la rime avec " Forrest Gump ", ce mot étranger est énoncé avec un plaisir particulier, qui explore des sonorités explosives. Tout en récupérant des idées de Norman Brown, José Miguel Wisnik suggère que

le langage « normal » semble toujours se soumettre à l'opérationnalité des signes comme l'union conventionnelle et stable entre le signifiant et le signifié, et la représentation. Dans la pratique, il est tout le temps harcelé par la mobilité primaire des pulsions qui le modifient en lapsus, en jeux de mots, en pures éclosions phonétiques, en projections imagées, qui décalent l'économie du signe linguistique vers des arrangements imprévus, et qui marquent la phrase par les ondulations dansantes du rythme corporel (Wisnik 1980: 3 ). 
Avant lui, Freud proposait déjà que la technique des jeux de mot (dimension que l'on peut considérer d'importance mais, pas exclusive des improvisations rimées), consistait à

Concentrer notre attitude psychique par rapport au son du mot plutôt qu'à son sens - faire en sorte que la présentation (acoustique) du mot prenne la place de sa signification, déterminée par ses relations avec les représentations des choses. On peut, avec raison, se méfier du fait qu'en le faisant on opère un grand soulagement du travail psychique, et qu'en utilisant les mots sérieusement, on s'oblige à un certain effort (Freud 1969: 117).

Quand Nino fait rimer " bang jump " avec " Forrest Gump », le non-sens du premier vers est compensé par la rime rare et l'évocation assez drôle de l'idiot qui, dans le film, ne fait que manger du chocolat, pendant qu'il raconte sa participation invraisemblable à de grands événements historiques. Remarquons aussi que Nino venait de mentionner le bonnet de pêcheur, ce personnage étant associé, dans l'imaginaire brésilien, au menteur, qui exagère quand il raconte ses prouesses au retour de la pêche. Y aurait-il une manière plus efficace d'attaquer quelqu'un qui est considéré comme une " légende vivante du freestyle brésilien » que de dire que c'est une farce, un mythomane compulsif?

Avant de poursuivre sur l'analyse des provocations de chacun, soulignons que " raconter des histoires drôles, dans la perspective anthropologique, est un discours direct, un genre performatif qui inclut des plaisanteries, des histoires, des contes populaires, des formes rituelles, des bouffonneries et de la conversation commune " (Driessen 2000: 254). De la même manière, les joutes de freestyle doivent aussi être pensées comme des performances. On a déjà vu que lors de ces disputes on ne parle pas n'importe comment: on s'exprime avec des rimes et du rythme. Plus précisément, les contours mélodiques du rap sont très proches de ceux du langage parlé, mais malgré tout, on l'identifie comme de la chanson. Sur ce sujet, Luiz Tatit suggère que « rien n'est plus radical, comme forme de chanson, qu'une parole explicite, qui annule les variations 'romantiques' de la mélodie tout en conservant l'intonation crue, sa matière première " (Tatit 2007: 231). Selon Adam Bradley

a rap verse is the product of one type of rhythm (that of language) being fitted to another (that of music). Great pop lyricists, Irving Berlin or John Lennon or 
Stevie Wonder, match their words not only to the rhythm of the music, but to melodies and harmonies as well. For that most part, MCs need concern themselves only with the beat. (BRADLEY 2009: 15).

Ce manque d'intérêt pour la mélodie fait en sorte que l'exploitation des divisions rythmiques devient la principale valeur musicale du chant-parlé, aussi bien dans les raps écrits que dans ceux improvisés " sur l'heure ". L'expression originelle de cette " façon » d'exploiter le rythme est flow - qui en anglais veut dire courant ou flux. C'est une métaphore pour la fluidité avec laquelle on espère qu'un improvisateur enchaîne ses rimes.

L'emploi de cette possibilité d'intonation est indispensable pour caractériser la performance des batailles et autoriser le fait de « se moquer des autres ". Dans l'ouvrage cité précédemment, Réflexions sur le ridicule et sur les moyens de l'éviter, l'auteur évalue que « la moquerie est d'usage difficile et même dangereux, si elle n'est pas accompagnée de circonstances qui retirent son " amertume " (Bellegarde apud Minois 2003: 446). L'emploi de la parole rimée et rythmée n'est pas sans importance dans la configuration de l'esprit moqueur et l'adoucissement, au moins partiel, de cette amertume à laquelle l'auteur fait référence. C'est ainsi que le " mauvais rire " peut devenir acceptable. C'est le cas du vers final de Nino, dans lequel il accélère le débit d'émission des mots tout en associant l'idée de flow à l'image d'une rafale de mitraillette. C'est ainsi qu'un vers comme: «j'arrache ta tête avec une carabine plutôt qu'avec un flingue " devient comique. D’après les coutumes dans les joutes, " celui qui a terminé doit recommencer " - et c'est alors Nino qui lance les premiers vers du deuxième round:

NINO

Alors ventripotent, viens sans faire chier

Avec Nino dans tes culottes tu vas te salir

T'es au courant, ici les choses sont différentes

Parce que Nino envoie des rimes intelligentes

C'est comme ça, ici il n'y a pas de phonique

Tu sais que t'as des dents aussi grosses que celles de Monique ${ }^{15}$

15 Mônica est un personnage de bande dessinée pour enfants, très connue au Brésil. C'est une petite fille qui a de grandes dents. 
Oui, tu sais, j'exagère pas, enfin

Putain, qu'est-ce que tes dents sont grosses, tu veux une carotte mon lapin?

Quand il s'agit de freestyle, Nino est le boss

Tu sais, mon joufflu, pour toi ça va être atroce

Oui d'accord, il faut que tu trouves un boulot

On va dire à tout le monde que tu es chauffeur de bus, putain que tu es gros

Ici à Santa Cruz, je vais tout compléter

Espèce de gros boudin débile, va te faire enculer!

Le deuxième round de Nino n'a pas eu le même effet sur le public à l'exception du vers " on va dire à tout le monde que tu es chauffeur de bus ". Lâge moyen des participants de bataille étant d'une vingtaine d'années, la plupart d'entre eux ont des emplois précaires tels que livreurs à moto, télémarketing ou bien des stages dans le cas de ceux qui accèdent à la faculté. La littérature a déjà traité amplement du thème du mépris envers le travail manuel, héritage d'une société basée sur l'esclavage ${ }^{16}$. Quand Nino suggère alors que « la légende » travaille comme chauffeur de bus, le public explose de rire, mais d'un rire un peu gêné.

Au moment de sa réponse, JL augmente le niveau de gêne du rire. Parmi les centaines de joutes auxquelles j’ai pu assister, jamais la limite du « mauvais rire " n’a été poussée aussi loin. En utilisant une expression commune, nous pouvons dire que JL a été très politiquement incorrect.

$J L$

J'ai regardé à côté et soudainement je ne regardais plus devant Mais mon frère, ça pue, tu pues des dents

Tu ne les brosses pas mon pote, c'est sauvage

Je me souviens bien, mais quand ils ont libéré les noirs de l'esclavage

ça fait chier que ce black soit sorti

Mais il n'est pas black, c'est qu'un pauv' type

En plus je ne vais pas vraiment dire ce que je voulais

16 Voir Wisnik 2008, entre autres. 
Tu dis que je suis rond mais tu es maigre comme un balai

Regardez-le, regardez que c'est moche

Pour ne pas voler il met des pierres dans ses poches

Les culottes que je porte, qui me les a offertes

Tu me les as offertes hier, tu sais pourquoi?

Dans un endroit quej'ai bien connu, j'ai halluciné

Des culottes marquées avec ton nom: pédé

JL fait comprendre qu'il regrette la fin de l'esclavage. Quand il ditt $^{17}$ : " mais quand ils ont libéré les noirs de l'esclavage ", on questionne tout d'abord la raison de l'utilisation de la préposition " mais ", qui suggère une certaine non conformité. Ensuite il spécifie: le problème n’est pas la libération des noirs, mais celle de Nino en particulier: « ça fait chier que ce black soit sorti ". J'ai eu l'impression que le public était gêné, mais personne ne s'est manifesté et la bataille a suivi son cours. JL semble se rendre compte qu'il touche à des sujets sensibles et essaie de s'en échapper, sans savoir trop comment: " mais il n'est pas black, c'est qu'un pauvre type ». Tout de suite après, il dévoile la pensée sur laquelle il a conduit son improvisation: " en plus, je ne vais pas vraiment dire ce que je voulais ». Il se rend compte qu'il ne peut pas continuer dans cette direction, qui le mènerait directement à la défaite: il ne peut pas dire ce qu'il voulait parce ce que ce qu'il voulait dire est une offense inacceptable dans le contexte des batailles. Il change alors rapidement de direction et répond aux provocations sur son poids en disant que son adversaire est trop maigre. À la fin du round, il récupère de manière confuse l'histoire des culottes, que Nino avait employée contre lui, et le traite de "gay ".

Même si la première partie de son improvisation a provoqué un certain malaise, JL finit par vaincre le deuxième round. Peut-être que ce qui explique sa victoire est le fait que s'il perdait le deuxième round, la bataille serait finie. Étant donné que c'était la première participation de « la légende JL » à Santa Cruz, il ne pouvait pas se faire éliminer dès la première étape. Voyons alors comment il tente de répondre au troisième round:

17 Même si ces événements sont déjà passés, pour des questions de style, je vais éventuellement utiliser le présent ethnographique. 
NINO

Tu as parlé des blacks, alors Marie Cendrillon

Venez tous les blacks, on va tabasser ce papillon

Tiens cousin, essaie de te faire une image

Tu sais que tu n'auras pas de massage ${ }^{18}$

Tu croises les bras, t'es gros, t'es pathétique

Tu ne fais penser ni à Zeca Pagodinho ni à sa musique

Ha, c'est comme ça, je suis monstrueux, moi

Nino est venu ici pour en finir avec toi

Tiens boudin, maintenant tu n'auras rien

Je ne suis pas un serpent mais j'ai du venin

Tu me vénères tu sais, je te shoote avec un gros flingue Glock

Comme Freddie Krueger,

Je te déchire espèce de boudin, enculé, idiot, débile

Nino n'a pas exploité la mention raciste de son adversaire. Avec le vers " venez tous les blacks, on va tabasser ce papillon » il a, en quelque sorte, répondu à l'offense de JL, mais sans savoir ou vouloir aller plus loin sur le thème du racisme. De ce fait, JL en profite pour enchaîner ces quelques rimes qui lui valent la victoire.

$J L$

Ceux qui ont écouté, maintenant faites attention à moi

Cinquante-six fois il a dit: tiens boudin, tiens boudin

Il a parlé de mon bide, de mon gros ventre

Mais t'as oublié de parler du ver solitaire qui est dans ton ventre, mon frère

Il $y$ a très longtemps que tu ne manges pas bien

Tu as dit quej'étais je ne sais pas quoi, tu n'en sais rien

Tu as eu peur de moi, aujourd'hui ou demain

18 L’expression « tu n'auras pas de massage », en brésilien, veut dire " ce ne sera pas facile pour toi ». 
Tu vas te fais virer du groupe É o Tchan (rires)

Franchement, je ne suis pas ton copain

Mais tes potes t'appellent Jacarè (rires)

Je danse un petit peu, JL est mon nom

Celui qui détruit Nino, t'as compris? c'est bon?

Mon pote, fais attention maintenant

Tu es balaise, je veux te serrer la main

Si au deuxième round JL a été peu habile (et c'est peu dire) quand il a improvisé sur le thème de la race, cette fois-ci il a mieux joué. Comparer son adversaire au célèbre danseur Jacaré du groupe bahianais "É o Tchan ", lui a valu la victoire. Ce qui est curieux, c'est que cette comparaison était une réponse à la toute première rime de Nino, qui l'avait comparé à Zacarias. Évidemment, ces plaisanteries ont fonctionné car JL est effectivement petit, rond et légèrement dégarni - comme Zacarias; et pour sa part, Nino a la peau très noire, de beaux traits et un corps plutôt athlétique, comme Jacaré. Marshall Sahlins nous explique le fait « qu'on ne peut pas insérer avec légitimité une 'voix' japonaise dans une ethnographie sioux " (Sahlins 2004: 521). De la même manière, il n'est ni amusant, ni efficace de dire que quelqu'un ressemble à quelqu'un d'autre si rien, que ce soit par identité ou par opposition, ne suggère cette comparaison. On se souvient alors que JL s'est aussi moqué du fait que son adversaire était mince. Mince ou bien athlétique? Il faut aussi considérer que la marge d'interprétation des attributs phénotypiques et leur transformation en objets de moquerie semble, malgré tout, être assez large.

La mention du groupe bahianais apporte une charge humoristique importante, étant donné leurs déguisements exotiques, leur manière peu conventionnelle de danser et leur musique exubérante, au paroxysme de la joie et de la provocation sensuelle. Tous les improvisateurs avec qui j'ai pu discuter ont été fermes en disant qu'aujourd'hui le rap « ne fait plus de distinction entre les blancs et les noirs - on a dépassé ça il y a très longtemps " (la phrase est de Marcello Gugu). Pourtant, comme nous l'avons observé, le thème réapparaît parfois de manière insidieuse. Ce qui est intéressant de remarquer, c'est la manière dont le repère de la race ou de la couleur ne devient « opérationnel » que lorsqu'il est associé à d'autres, de manière 
très spécifique. C'est à dire que quelques articulations fonctionnent bien, d'autres moins bien- sachant que 'fonctionner', ici, veut dire provoquer le rire et l'effet d'adhésion. C'est une évidence du fait que, comme disait Freud, « toute plaisanterie a besoin de son public: partager le rire devant les mêmes histoires drôles est une évidence d'une grande conformité psychique " (Freud 1969: 144). Au moment d'analyser les joutes, on peut chercher à reconstruire quelques-unes des hypothèses partagées ${ }^{19}$ par le groupe, qui sautent aux yeux, si on remarque l'efficacité de certaines articulations et l'échec de certaines autres. Il faut considérer aussi que ces hypothèses partagées ne sont pas figées et doivent être constamment revues - c'est là l'intérêt des joutes. Quand JL compare son adversaire au danseur de «É o Tchan ", il dit que sa beauté, sa peau très noire et son physique athlétique sont drôles, en opposition à la petite taille, la rondeur et la laideur de Zacarias. Malgré le fait que beauté et force physique puissent être des qualités en principe positives, elles deviennent risibles lorsqu'elles se prêtent à une danse sensuelle et exotique, sexuellement ambiguë. Il semble certain que la couleur de la peau est le trait central mobilisé, surtout si l'on en considère le round précédent. Mais il ne serait pas suffisant s'il n'était pas lié à d'autres éléments.

Ces attributs sont valables, car ils sont utilisés non pas comme une simple moquerie, mais en tant que représentation de catégories sociales qui deviennent significatives dans la mesure où elles opèrent comme des « repères sociaux de la différence ", articulés " dans des systèmes de classification régulés par des conventions et des normes. Ces catégories n’acquièrent pas de sens uniquement isolées les unes des autres, mais surtout à travers la connexion intime qui s'établit entre elles (...) En fait, ces repères se prêtent à établir des relations de relations " (Schwarcz et Starling 2005: 219) ${ }^{20}$. Cette suggestion théorico-méthodologique nous permet de gérer le matériel des

\footnotetext{
19 Je tiens à remercier Lilia Schwarcz pour la suggestion de l'expression.

20 Livio Sansone propose la même pensée quand il affirme que « la perception de la race et de l'identité ethnique, loin d'être universelle, est médiatisée par la classe, la génération, le métier, la position géographique et le genre " (Sansone 2004: 255). Une autre proposition intéressante est celle de Manuela Carneiro da Cunha, selon qui « l'identité est construite de manière situationnelle et contrastante, c'est à dire, que c'est une réponse politique à une configuration, une réponse articulée avec d'autres identités qui sont aussi en jeu, avec lesquelles elle forme un système. C'est une stratégie de différences " (Cunha 1985: 206). Voir aussi le texte de présentation du NUMAS (Centre d'études sur les repères sociaux de la différence), rédigé par Julio Simões et Lilia Schwarcz et disponible sur le site http://numas-usp.blogspot.com
} 
improvisations de manière articulée, sans, pour autant, « fixer » des catégories comme des stéréotypes qui se répéteraient de façon continue.

Passons alors à un autre cas. Au cours des premiers mois de l'année 2009, une seule jeune fille a participé assidûment aux duels en tant qu'improvisatrice. Si nous nous basons sur le volume de " retour sonore " émis par les spectateurs, on peut affirmer que les batailles livrées par Thais Pri ont été celles qui ont le plus enthousiasmé le public (qui votait soit pour elle, soit contre elle). Admettons que cette indication indigène soit significative et prenons comme exemples deux batailles réalisées à deux semaines d'écart, au cours desquelles Pri s'est battue contre un jeune homme connu sous le nom de Basthian.

Voici une sélection de vers du premier round de la bataille du 6 juin 2009:

BASTHIAN

c'est le début de la bataille, je frappe Thais Pri

bien sûr, il faut rester calme, attends, je t'en prie

je vais déchirer cette fille

t'as de la chance qu'il ne pleuve pas, t'as pu te faire un brushing (rires)

mec, je vais continuer sous ce ciel nuageux

si tu avais fait un brushing il serait déjà devenu un tas des dreads

c'est sûr, laisse moi te dire

t'as de a chance que ton brushing ne soit pas devenu Black Power

j'envoie grave, c'est sûr, c'est le premier round et je déconne

La première stratégie de Basthian a été d'utiliser celui qui est peut être le trait phénotypique le plus mobilisé en tant que repère de distinction de race au Brésil: les cheveux. Il suffit de penser à la célèbre chanson " Mulata ", ${ }^{21}$ écrite par les frères Vitale dans les années 20, et jusqu'à nos jours chantée dans les bals de carnaval malgré son contenu fortement raciste:

21 Quelques années plus tard, Lamartine Babo a repris et modifié cette chanson pour la relancer à l'occasion du carnaval carioca sous un nouveau titre, "O teu cabelo não nega » (« Tes cheveux ne laissent pas nier"). 
Tes cheveux ne nient pas, Mulata

Parce que ta couleur est métissée

Mais étant donné que la couleur ne se transmet pas

Alors Mulata, je veux t'aimer ${ }^{22}$

Le refrain détient une contradiction assez curieuse: le protagoniste est attiré sexuellement par la métisse (" Mulata »), mais il ne souhaite pas « attraper " sa couleur. Quelque part, cette couleur de peau est en même temps source d'attraction et de répulsion. Les cheveux, par contre, sont " mauvais " quoiqu'il arrive. L'hypothèse semble être confirmée par Livio Sansone, quand il affirme que

D’après la norme somatique hégémonique dans les classes populaires, à Bahia, comme on le voit dans la littérature de cordel, la personne qui a la peau claire et les cheveux frisés est considérée moche et potentiellement perfide; tandis que la personne qui a la peau très foncée et les cheveux naturellement lisses peut être belle, parce que soit disant elle aurait la beauté de la peau noire et la 'finesse' de cheveux fins naturels. Un dicton très connu selon lequel 'la couleur, on la reconnait par les cheveux derrière la nuque' (la partie 'cachée' des cheveux, celle qui ne peut pas être lissée). (Sansone 2004: 256).

Lilia Schwarcz a recueilli un autre témoignage sur ce sujet et qui vaut en tant que règle pour classer les joueurs lors du match annuel de football du bidonville d'Heliópolis, à São Paulo. Connu comme le match des « blancs contre les noirs ", la sélection des équipes est en soit un exercice curieux. On dit que « si les cheveux balancent, le sujet ne peut pas jouer pour les noirs " (Schwarcz 2007: 240). Pensons alors au cas du métro de Santa Cruz. À l'époque de cette bataille, Pri s'était fait lisser les cheveux par un brushing. On ne peut pas savoir si ses cheveux naturels étaient peu ou très frisés ${ }^{23}$, mais il est évident qu'elle les avait lissés et son adversaire ne s'est pas privé de l'exploiter de deux manières. D’abord, il s'est moqué du fait qu'elle a de « mauvais » cheveux, qui « deviennent des dreadlocks s'ils prennent la pluie ». Mais

22 O teu cabelo não nega, Mulata

Porque és mulata na cor

Mas como a cor não pega, Mulata

Mulata quero o teu amor

23 Au Brésil on utilise plusieurs termes, la plupart d'entre eux de manière dépréciative, pour désigner les cheveux frisés. Quelques-unes des plus courantes sont " cheveux mauvais " (cabelo ruim) et " cheveux durs " (cabelo duro). 
en même temps, il la critique de les avoir fait lisser, de vouloir cacher ses cheveux naturels. On ne peut alors savoir ce qui est le pire: les cheveux de Pri deviennent une contradiction sans solution.

Le public a réagi avec enthousiasme aux rimes de Basthian, mais il faut reconnaître qu'il y a eu une certaine gêne quand il a dit « t'as de la chance que ton brushing ne soit pas devenu Black Power ». Ce n'est pas pour rien qu'il a été obligé de rajouter une phrase qui n'a pas rimé, et qu'il est en plus sorti de la quadrature. L’intention vocale était claire: il pensait conclure son improvisation avec « Black Power »; mais à la dernière minute quelque chose l'a poussé à dire encore quelques mots, comme une demande d'excuses: «j'envoie grave, c'est sûr, c'est le premier round et je déconne ". On peut facilement interpréter ce vers comme un essai d'assouplir la proposition précédente. Comme s'il comprenait qu'il ne fallait pas trop insister sur le thème des cheveux, en utilisant en plus une expression positive comme « Black Power ", à l'origine de tout un mouvement social. On verra plus tard comment la répétition de cette plaisanterie l'obligera catégoriquement à s'expliquer.

Pri répond de manière assez efficace, le public s'emballe et finit par la choisir comme gagnante du premier round.

PRI

J'envoie mes rimes et toi tu désespères

Tu es MC ou bien coiffeur? (rires)

Espèce de traître, sois un peu plus sage

Tu t'occupes trop de mon image

Va t'occuper de la tienne, est-ce que tu t'es déjà regardé dans une glace?

Tu es trop moche, espèce de gamin, tu n'es qu'un lâche (rires)

Tu sais, je suis prête à te combattre

J'arrache ta petite tête et tes petites jambes de plâtre

Fais attention à mes rimes

Je mets une bonne ambiance, toi tu la mines

Je te déchire, enculé, il faut que tu t'y fasses

Ici je vais gagner tout l'espace 
Dans ses premiers vers, Pri affirme que le véritable MC ne doit pas s'occuper des questions d'esthétique, mais de la qualité de ses rimes et de son engagement sur le rap. C'est pour cela que le public a réagi avec autant d'enthousiasme quand elle a dit: « tu es MC ou bien coiffeur? » Il faut souligner le fait que le choix de l'image du coiffeur fait référence à l'homosexualité. Basthian est questionné sur ces deux domaines: est-il un véritable MC? Est-il homosexuel? Peut-on être en même temps homosexuel et MC? ${ }^{24}$

Dans la suite de son improvisation, Thais Pri utilise des images agressives, de véritables menaces verbales contre son adversaire. Au moment où elle se dit " prête à le combattre » et à lui « arracher la tête ", les spectateurs s'emballent. Quelqu'un fait un commentaire pour l'encourager à poursuivre: " elle est pleine de révolte ». Le deuxième round aura pourtant moins d'impact sur le public:

PRI

J'envoie mes rimes en utilisant mes critères

Je vais envoyer cet imbécile directement au cimetière

Ecoute mon frère, l'humilité est de naissance

Mais tu sais qu'avec toij'ai toute mon aisance

Tu n'es qu'un squeletton, espèce de sous humain

Tu n'envoies que des rimes profanes

Avec ta petite moustache pleine de poux

Mets-la au bain marie, quel dégoût

Parce qu'ici mon frèrej'envoie les rimes et tu vas avoir froid

Prends garde parce que tout le monde ici me préfere à toi

Tes rimes sont restées à moitié

Malheureusement tu ne fais qu'essayer

Tu nous fais tous avoir de la peine, tu ne fais qu'essayer

Tu viens ici et tu me regardes, tu ne fais que m'admirer

24 Depuis le début des années 200o, le mouvement appelé 'Homo-Hop' prend de l'ampleur, notamment grâce au travail de MCs homosexuels assumés, comme Deadlee et Foxxjazell qui critiquent dans leurs paroles les diffêrentes formes d'homophobie. 
Parce que je fais des rimes mieux que toi

Quand tu rimes toi tu n'es pas capable de causer l'effroi

À son tour, ayant perdu le premier round, Basthian ne peut perdre cette opportunité. Pour le moment, il ne peut traiter du discours sur les cheveux lissés ou raides. Parler de ce sujet serait pris comme « une affaire de coiffeur " et non pas de $M C$, mais il récupère la thématique de manière habile, en disant qu'il allait détruire la fille ébouriffée.

\section{BASTHIAN}

Etre MC est une profession et toute profession a une éthique

T'as dit que je dis des conneries mais c'est toi qui ne rimes que sur l'esthétique

Merde alors: fais très attention à ce que tu as dit

T'as dit quej'étais moche mais personne n'a ri (rires)

Je vais suivre habilement, je te dis

Je vais en finir avec cette petite fille qui pense qu'elle est un vrai $M C$

Tu n'es pas underground, tu ne connais rien au freestyle, je te prie

Qui es-tu? Tu n'es pas MC, tu n'es que Thais Pri

Je déchire cette fille ebouriffée au milieu du trottoir

Au milieu de la nuit, personne ne peut avec Basthian

Je suis agressif comme le Wu Tang Clan ${ }^{25}$ (bruits)

Lors de ses derniers vers, Basthian a accéléré le débit et le volume d'émission des mots, faisant monter la tension et la sensation de violence, comme s'il était vraiment prêt à se battre physiquement. Les commentaires des spectateurs confirment l'efficacité de sa stratégie: « Agressif mon frère! »; « Vénère! » Et c'est ainsi qu'il arrive à gagner le deuxième round.

Dans sa dernière improvisation, Basthian reprend une provocation précédemment utilisée, quand il traite Pri de " petite fille ». Il est probablement au courant qu'il prend le risque de se faire accuser de machiste, mais il insiste quand même.

25 Groupe de rap new yorkais dont l'apogée de sa popularité a eu lieu pendant les années go. 
BASTHIAN

Je commence habilement

Je rime sur le trottoir maintenant

Toi comme toutes les femmes tu veux plus de place

Alors je vais te donner une cuisine plus grande pour que tu t'y fasses (rires)

C'est juste une plaisanterie, des rimes avec de l'adrénaline

Mais je ne vais rien dire

Je vais en finir avec ce féminisme

C'est des rimes ici, putain, le freestyle j'en ai des listes et des listes

Tu as parlé d'esthétique tout le temps

Va travailler dans un salon, putain

Tu n'es pas faite pour le freestyle, tu apprends tes rimes dans le dictionnaire

Ou bien va voir des joutes sur internet pour les apprendre par cœur

Tu peux être sûre, je vais t'expliquer

Un vrai $M C$ quand il fait des rimes devant la glace il peut l'exploser

Lors du premier round Basthian s'était excusé d'avoir fait une blague sur les cheveux " black power » de son adversaire. Ici il utilise le jargon machiste comme quoi « les femmes appartiennent à la cuisine ${ }^{26}$ et ensuite explique que c'est juste une plaisanterie. Pourquoi se sent-il obligé d'expliquer que ce n'est qu'une plaisanterie, alors que tout le monde sait que les joutes sont justement faites pour ça: une occasion de taquiner l'autre et de rire? Aussi bien la provocation sur les cheveux que la phrase machiste sont, aujourd'hui, " politiquement incorrectes " au Brésil. On peut assumer que dans les hypothèses partagées avec le groupe, ce sont des propos dangereux. Alors Basthian évalue son risque et préfère rajouter un petit mot d'excuse. Ce qui est particulièrement pernicieux c'est que, tout en reconnaissant le fait que ce sont des propos réactionnaires, Basthian choisit de les utiliser. Et il considère qu'un petit mot d'excuse suffit à l'autoriser à le faire. Un autre point à noter est que, tout comme JL, après avoir énoncé quelques propos racistes (ou machistes)

26 Référence à un dicton brésilien machiste, selon lequel " la place de la femme est à la cuisine " (« lugar de mulher é na cozinha "). 
il rajoute: « mais je ne vais rien dire ». Il faut donc croire qu'il y a beaucoup de non-dits qui ne sortent pas, car ils feraient l'effet d'une bombe. Et malgré tout, il y a des règles à respecter lors de ces joutes.

Encore dans le but de se protéger et tout en attaquant, Basthian transforme la " petite fille » du round précédant en une féministe. Mais il introduit aussi un nouveau thème: celui de la valorisation de l'improvisation. Aucune accusation ne peut être plus grave pour un freestyler que celle d'apprendre des rimes " par cœur ». Peter Burke, par exemple, nous rappelle le fait que le terme 'improvisation' est plus ambigu qu'il n'en a l'air, "parce que la dichotomie entre l'improvisé et le mémorisé est fausse. N’importe quel orateur sait qu'il existe toute une gamme de possibilités entre les deux extrêmes de quelque chose de mémorisé ou bien quelque chose de créé sur le moment, sans préméditation " (Burke 1989: 169). D’après le récit des freestylers, l'une des principales stratégies pour se développer en tant qu'improvisateur est justement d'apprendre à étendre son vocabulaire (par exemple en « apprenant des rimes dans le dictionnaire »). Le talent étant celui d'articuler les rimes de façon à faire croire que l'on improvise - et, pour cela, il existe des stratégies telles qu'utiliser des références « du moment » et d'exploiter les possibilités rythmiques de scansion.

Il faut aussi noter que la précarité de la composition du rap (qu'on ne peut pas huer) explique l'importance centrale de la création des textes ${ }^{27}$. C'est ce que dit Sami Zegnani:

Une des particularités du rap, par rapport aux autres musiques contemporaines " jeunes », réside dans la revendication de la paternité des lyrics par les artistes. Nous voici confrontés à un style musical où les auteurs et les interprètes sont les mêmes personnes. Chanter - au sens classique du terme - et rapper ne peuvent être approchés de manière analogue, car ne pas être auteur des textes que l'on rappe provoque le blâme et l'exclusion quasi certaine du mouvement hip-hop. (Zegnani 2004: 65).

L'accusation de rimer par cœur ou bien de rimer des vers écrits par quelqu'un d'autre, est courante à Santa Cruz, ce qui renforce l'hypothèse de Zegnani.

27 Dans d'autres genres de chanson populaire, on donne souvent plus de valeur à l'interprète plutôt qu'à l'auteur ou le compositeur. Les artistes de rap ne vont jamais, ou très exceptionnellement, chanter des paroles qu'ils n'ont pas écrites eux-mêmes. 
Deux semaines plus tard, le 20 juin, Basthian et Pri se sont à nouveau défiés. Quand le présentateur Pivete les a vus à nouveau face à face, il a lancé avec enthousiasme: « C'est mortel! Peut être alors qu'on verra la revanche! »

PRI

J'envoie la rime qui prend toute la place sur le plateau

Machiste de merde, je vais te tuer avec un couteau

Tu sais que je suis bonne à l'improvisation, plus qu'une minette

Alors je vais te tuer avec une mitraillette

Tu auras plein de petits trous, comme un con

$\mathrm{Tu}$ sais que ton freestyle est tout saufbon

Attention, le beat n'est pas assez fort

Mais, quand même, moij'ai raison et toi tu as tort

Tu as tort et aujourd'hui tu auras du mal

Ici dans cette bataille je vais gagner, c'est normal

Tu sais c'est comme ça que j'envoie

Tu n'es qu'un salaud, je vaux bien plus que toi

... (Pri s'embrouille avec le beat et murmure des syllabes sans aucun sens)

Tu sais tu n'envoies que des vers de dandy

BASTHIAN

Je suis un homme pour de vrai, je ne suis pas machiste

C'est pas de ma faute si tu as tes règles, espèce de féministe

Ca ne te plait pas une cuisine plus grosse, laisse moi te dire,

Alors que c'est la laverie que je vais t'agrandir

Petite fille qui croit représenter toutes les nanas

Tu n'as pas de flingue, alors vite bouge de là

Basthian en finit avec toi, quelle saloperie

$T u$ n'es pas MC, pour toi les rimes ne sont qu'une plaisanterie 
Tu restes sur internet, sur youtube, en train de déconner

"Je dois apprendre le freestyle des autres et tout mémoriser "

On peut observer que les mêmes catégories et stratégies de rhétorique de la bataille précédente ont été reprises. Notamment l'opposition machiste contre féministe semble être bien établie. Ce jour-là, un des organisateurs avait apporté une chaîne hi-fi, avec laquelle il lançait des bases musicales. Etant donné que le système de sonorisation était minimal, le volume était très faible et il fallait faire très attention à ne pas " croiser " le rythme. Pri a eu un peu de mal avec le beat et a fini par se confondre dans les rimes. À la fin de son improvisation, elle rit un peu du fait de s'être emmêlée. Basthian insiste en la provoquant sur des thèmes sexistes: d'abord en disant que c'est " un homme pour de vrai ». Il s'oppose aux "faux hommes " (le coiffeur, le dandy) et en même temps à la femme, tout en se protégeant: " je ne suis pas machiste ». Ensuite, il retourne sa proposition et attaque: " c'est pas de ma faute si tu as tes règles, espèce de féministe ". Alors, Pri est encore une fois ciblée d'une double attaque: c'est une femme (qui a ses règles et ses humeurs, d'ailleurs une thématique courante dans les discours machistes), mais serait, en plus, féministe (une fausse femme). Le même type de double faute qu'il avait utilisée pour attaquer ses cheveux drus lissés. Après avoir dit qu'il n'était pas machiste, Basthian n'hésite pas à dire: « ça ne te plaît pas une cuisine plus grande, laisse-moi te dire, alors que c'est la laverie que je vais t'agrandir ". Pour finir, il l'accuse à nouveau d'apprendre des rimes par cœur, de rimer par plaisanterie et, de ce fait, de ne pas être une MC.

C'est Basthian qui gagne ce round et le public est très enthousiasmé. Deux des spectateurs échangent des propos rassurants: « je voulais dire que je ne suis pas machiste ». L'autre est d'accord: " personne ici n'est machiste "; alors le premier renchérit: " il ne faut pas être machiste ".

Cette dénégation du machisme est particulièrement intéressante si on la rapproche de la dénégation du racisme dont nous avons parlé précédemment. On entend des échos d'une phrase citée par Florestan Fernandes: « nous, les brésiliens, avons le préjugé d'avoir des préjugés ». Cette ambiguïté, si particulière, est propre aux préjugés de couleur ainsi qu'à celui du genre; ce sont des phénomènes qui veulent toujours s'échapper, comme des poissons vivants. Il est certain que la couleur et le sexe ne font aucune différence...même s'ils en font quand même. 
Au deuxième round, la bataille de Pri et Basthian a incorporé de nouveaux éléments. Thiagão, un MC plus âgé et expérimenté, s'est placé à côté du présentateur Pivete, et a joué un rôle de " clown ", pour donner du souffle aux improvisateurs. Il crie alors: « Sois méchant, Basthian, fais-moi voir! »

\section{BASTHIAN}

J'y vais, méchant, habilement je descends le bidonville

Basthian détruit la petite fille et laisse son corps par terre dans le bidonville

Tout le monde le sait, t'as qu'à te taire

Tu n'es qu'une bourgeoise, protégée par le maire

Je viens du bidonville et jamais le maire n'est venu nous voir

Putain, je voulais le rap dans les parages et il a fallu le faire moi-même

J'ai dû créer moi même un événement pour qu'on puisse rimer

Et maintenant, je dois entendre cette petite fille que je ne peux pas blairer

Quelle tristesse, je n'en peux plus

Tes rimes sont mauvaises, petite fille je te tue

Le public est très excité et crie des phrases comme: " feu à Babylone! »; déchire-le avec une mitraillette! »; « explose sa race! »

PR1I

Je n'envoie que de bonnes rimes, pour de vrai

Tu viens du bidonville, c'est pour ça qu'elle reste ce qu'elle est

Mon frère, bourgeois c'est mon cul

Je viens d'Itapevi pour t'exploser parce que tu pues (bruit)

Ecoute-moi, tu es lent

Tu sais très bien que tu n'as aucun talent

Alors tu rimes mais tu n'as pas d'idées, aucune

$\mathrm{Tu}$ dis que les femmes ne valent rien mais ta mère en est une

Le public fait tellement de bruit qu'on n'entend plus ce qu'elle dit. Elle continue encore pendant quinze ou vingt secondes mais personne ne 
l'entend. Santa Cruz est en feu. Quelqu'un dit à Pri pour la taquiner: « du calme, du calme, il faut respirer et ensuite continuer. La nana est énervée. » Une autre fille se montre solidaire: «Putain, personne ne lui a laissé finir ses rimes, c'est quoi ça? » Thais Pri elle-même s'est un peu plainte: « merde alors, j’ai envoyé plein de rimes super, vous n'entendiez pas ». Quand le présentateur a demandé du bruit, Pri a été acclamée et a largement gagné le deuxième round. Quelqu'un dit: "ça, c'est un knock-out ».

On voit un nouveau thème apparaître dans l'agenda de nos improvisateurs: classe sociale. Nous ne souhaitons pas ici discuter le concept même de classe sociale - alors acceptons les termes indigènes employés par opposition: bidonville contre bourgeois. Le premier semble avoir une connotation positive tandis que le deuxième a l'air d'une grave offense. Basthian construit un argument basé sur l'idée d'appartenir au bidonville, d'être un véritable MC et de se battre pour le rap. Pri répond en disant qu'elle vient d'Itapevi, ville très pauvre de la banlieue de São Paulo - son origine géographique sert alors en tant que repère pour nier une accusation de classe sociale élevée. Ensuite, elle répond à l'argument sexiste: « tu dis que les femmes ne valent rien mais ta mère en est une ». Les réactions sont tellement enthousiastes qu'on n'entend plus rien. Ces mots ont assurés la victoire de Pri pour cette étape, mais on se demande si dans cette réaction, il n'y avait pas aussi l'envie de la couper dans ses mots. Thiagão dit, très animé: « cette bataille devient une vraie référence ! ».

On a vu comment les catégories de genre, de classe et d'origine géographique se sont articulées dans les improvisations. Le caractère relationnel de ces " repères de distinction " va émerger de manière très évidente lors du troisième et dernier round:

PRI

J'envoie des rimes, je rime jusqu'à l'aube

J'ai de la peine pour ta mère qui a mis au monde cette daube

Elle a attendu et attendu

Elle s'est dit « c'est con d'avoir attendu » quand t'es apparu

Mon frère tu sais bien que tu es le pire

Je vais à la cuisine mais c'est pour te faire cuire 
Tu dis que tu es du bidonville, mon cul

T'es pas malin,j'ai parlé et tu t'es tu

Tu n'es pas du bidonville, tu n'es qu'un bourgeois

Alors fais attention, tu ressembles à une oie

J'évolue et toi non, tu vas perdre, je te jure

Tu n'es pas du bidonville... si tu l'étais tu serais en train de voler des voitures

Avant d'acclamer ce dernier vers, Pri s'est montré peu satisfaite et on pourrait même croire qu'elle le regrette. La dernière phrase a été dite sans le tonus du reste de l'improvisation, sans faire attention aux divisions rythmiques, presque en faisant la grimace. Le public a tout de suite commencé à critiquer: " mais ça ne va pas ça, c'est un préjugé »; " c’est dommage, elle avait si bien commencé "; " Là, elle s'est trompée sur le dernier vers ». On peut considérer que les freestylers font partie d'une communauté de valeurs - les improvisations seraient alors des pratiques qui recadrent ces valeurs partagées. Le succès de l'improvisateur serait donc lié à sa capacité de faire rire, en articulant des catégories dans un discours performatif. La dernière plaisanterie de Thais Pri n'a pas été drôle pour le public, mais prise comme une offense. Alors, Basthian était déjà vainqueur de la bataille quand il a dit ces vers finaux:

\section{BASTHIAN}

Oui ma mère est une femme et elle a pu tomber enceinte

Toi, pourtant, tu ne vas jamais tomber enceinte parce personne ne t'aime (bruits)

Qu'est-ce que tu racontes, commence tout de suite à pleurer

Si tu tombes enceinte, après tu vas avorter

(Les spectateurs n'approuvent pas ce vers et font la moue)

Basthian continue à détruire la petite fille

Il va la laisser toute rouge dans la salle de chirurgie

Dégage, tes rimes sont pas d'enfer

Dégage petite fille aux cheveux de paille de fer

(Nouvelle réprobation du public)

C'est juste pour déconner

Je suis du bidonville mais je n'ai pas besoin de voler 
Je peux très bien travailler

Je ne vais pas me soumettre, je vais arriver au sommet et m'y accrocher

Avant de demander de faire du bruit, le présentateur Pivete se plaint auprès de Basthian: " il faut arrêter avec ce truc des cheveux frisés "! Basthian savait qu'il s'agissait d'un thème délicat, c'est pour cela que, juste après sa provocation, il a souligné, encore une fois, que c'était « juste pour déconner ». En tout cas, après avoir entendu la plainte de Pivete, Basthian a enlevé sa casquette et a montré ses cheveux, tout en disant: « les miens aussi sont frisés ».

L'hypothèse proposée dans cet article est que, dans les joutes de freestyle, les éléments poétiques et musicaux contribuent à ce que la moquerie soit acceptable - même si, quand ils formulent des catégories distinctives, les improvisateurs parfois se sentent obligés de renforcer que ce ne sont que des plaisanteries. Pour conclure, notons un fait amusant: quelques semaines après ces duels, Pri et Basthian ont commencé une histoire d'amour.

\section{BIBLIOGRAPHIE}

ABRAHAMS, Roger. 1970. Deep Down in the Jungle - Negro Narrative Folklore from the Streets of Philadelphia. Chicago: Aldine Publishing Company. ANDRADE, Mario de. 1989. Dicionário Musical Brasileiro. São Paulo: EDUSP. BRADLEY, Adam. 2009. Book of Rhymes-The Poetics of Hip Hop. New York: Basic Civitas.

BROWN, Joseph Edward. 2006. How to Freestyle, Write Battle Raps, and Write Rap Songs. Publicação independente.

BURKE, Peter. 1989. Cultura Popular na Idade Moderna. São Paulo: Companhia das Letras.

CUNHA, Manuela Carneiro da. 1985. Negros, Estrangeiros. São Paulo: Brasiliense.

DRIESSEN, Henk. 2000. "Humor, riso e o campo: reflexões da antropologia”. In: J. Bremmer \& H. Roodenburg (orgs.), Uma História Cultural do Humor. Rio de Janeiro: Record. pp. 251-276.

FREUD, Sigmund. 1969. Os Chistes e sua Relação com o Inconsciente. Obras completas. Rio de Janeiro: Imago Editora. 
GAY, Peter. 1995. A Experiência Burguesa da Rainha Vitória a Freud. v. 3. O Cultivo do Ódio. São Paulo: Companhia das Letras.

HUIZINGA, Johan. 2008. Homo Ludens: o Jogo como Elemento da Cultura. São Paulo: Editora Perspectiva.

MINOIS, Georges. 2003. História do Riso e do Escárnio. São Paulo: Editora da UNESP.

OLIVEIRA, Allan de Paula. 2007. "Quando se Canta o Conflito. Contribuições para a Análise de Desafios Cantados." Revista de Antropologia, 5o(1): 313-345. ROSE, Tricia. 1994. Black Noise: Rap Music and Black Culture in Contemporary America. Middletown: Wesleyan University Press.

SAHLINS, Marshall. 2004. Cultura na Prática. Rio de Janeiro: Editora UFRJ. SALIBA, Elias Thomé. 2002. Raízes do Riso. São Paulo: Companhia das Letras. SANSONE, Livio. 2004. Negritude sem Etnicidade: o Local e o Global nas Relações Raciais e na Produção Cultural Negra do Brasil. Salvador/Rio de Janeiro: Pallas. SCHWARCZ, Lilia Moritz. 2007. "Pretos Contra Brancos - ou Dando e Mudando Nomes”. In: J. P. Cabral e S. Viegas (orgs.), Nomes: Gênero, Etnicidade e Familia. Lisboa: Almedina. pp. 219-244.

SCHWARCZ, Lilia e STARLING, Heloísa. 2005. "Lendo Canções e Arriscando um Refrão". Revista USP, 68: 210-229.

TATIT, Luiz. 2007. Todos Entoam. São Paulo: Publifolha.

TOOP, David. 2000. Rap attack 3: African Rap to Global Hip Hop. London: Serpent's Tail.

WISNIK, José Miguel. 1980. Dança Dramática (Poesia/ Música Brasileira). Tese de Doutoramento, Universidade de São Paulo - São Paulo.

WISNIK, José Miguel. 2008. Veneno Remédio. São Paulo: Companhia das Letras.

ZEGNANI, Sami. 2004. "Le Rap Comme Activité Scripturale: l'émergence d'un Groupe Illégitime de Lettrés”. Langage et Société, 110: 65-84. 


\section{À propos de l'auteur}

Ricardo Indig Teperman

Departamento de Antropologia Social - FFLCH USP

Principaux ouvrages:

A Torcida Grita (CD) - Cultura Marcas, 2007.

Geringonça (CD) - O+ Music 2009.

Adresse:

Rua Dr. José Almeida Camargo, 155

05436-040 Jardim das Bandeiras

São Paulo - SP

email: ricardoteperman@yahoo.com.br 